\title{
Modernization of Control Systems of Electric Drives of Mine Lifting Machines
}

\author{
Ziyodullo Eshmurodov ${ }^{1}$, and Folib Holboiv ${ }^{2}$ \\ ${ }^{1}$ Navoi State Mining Institute, Uzbekistan \\ ${ }^{2}$ Navoi Mining and Metallurgical Combine, Uzbekistan
}

\begin{abstract}
The article shows the peculiarity of the system of technological automation of the newest Simatic S7-1500 PLCs, for electric drives of hoisting transport machines, the analysis of control systems for electric drives of operating mine hoisting machines of mining complexes. One of the main ways to increase the energy efficiency of MLM is to replace old asynchronous electric motors with a phase rotor with squirrel cage induction motors designed for operation in a frequency-controlled electric drive. The introduction of a frequency converter and an electric drive control system, which together allow the recovery of electricity in the supply network in the mode of generator braking. Thus, the system for automated control of the mine hoisting machine based on the Simatic S7-1500 PLC, with the developed control algorithm, is an integrated energy-efficient control system, which it is advisable to use in the management of the SHM.
\end{abstract}

\section{Introduction}

The electrical equipment of hoisting transport machines (HTM) operates under difficult conditions, connected with dustiness of air, vibrations, in some cases with high temperature and high humidity. The operating mode of the electric drive systems is intensive repeated short-time with frequent starts, reverses and braking.

The analysis of the existing electrical equipment of mining complexes has shown quite a wide application of asynchronous motors with a phase rotor (AMPR) for powerful (more than $300 \mathrm{~kW}$ ) mechanisms with severe starting conditions (EP of hoisting and transport mechanisms, EP conveyors, etc.). To date, the management of the production process based on the data of electromechanical systems is carried out by introducing additional active resistances into the rotor circuit of the motor. This way of regulation in our time has exhausted itself due to energy inefficiency $[1,2]$.

Currently used parametric control systems for starting and adjusting the speed of the AMPR are uneconomic and do not meet modern requirements for dynamic indicators.

One of the main ways to increase the energy efficiency of ShME is to use electric motors with a squirrel-cage rotor (Figure 1.), designed to operate as part of a frequency-controlled electric drive. The introduction of a frequency converter and an electric drive control system, which together allow the recovery of electricity in the supply network in the mode of generator braking.

Thus, the task of developing and researching an energy-efficient electric drive of the ShPM, with the possibility of electricity recuperation, is topical. 


\section{Analysis of the state of electric drives}

For today, at the mining enterprises there is a process of transition from old control systems to more perfect ones. Since the main consumers of electric power in industry are electric drives, this process acquires an urgent scientific task. In Fig. the block diagram of the electric drive of hoisting machines is given.

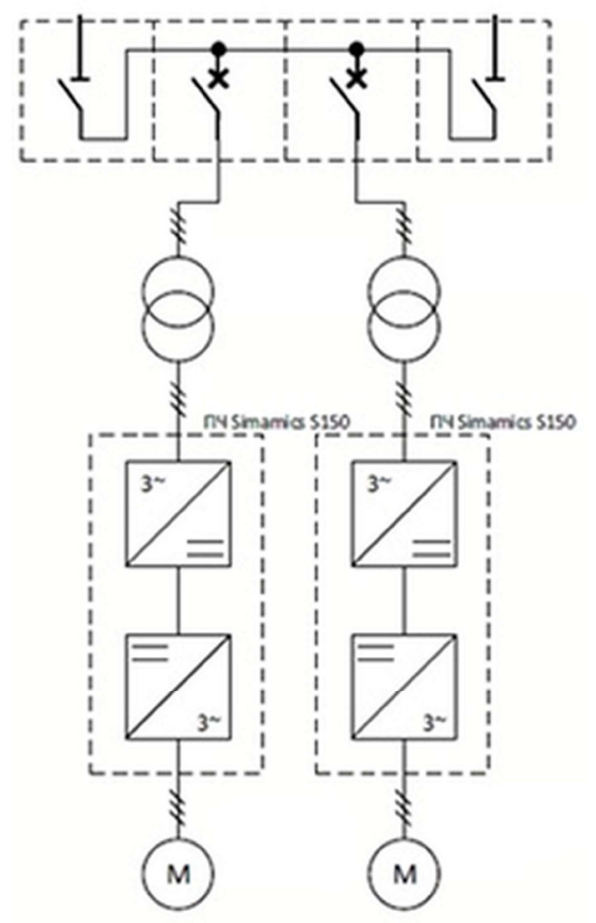

Fig. 1. Structural diagram of the electric drive of lifting machines.

In asynchronous motors, reinforced insulation of the windings, calculated for a voltage of $6 \mathrm{kV}$, is used, the motors are equipped with impulse encoders and fans of the "rider" type. The motors are made on rolling bearings with grease, the bearings on the non-drive side of the shaft are insulated.

The motor is reversible. The adjustment of the speed of rotation from zero to the main one is made by changing the input voltage and frequency.

The motor is powered by a frequency converter with a DC voltage link with pulse-width modulation.

The lifting machines are equipped with thyristor frequency converters (inverters) Siemens SINAMICS S150. The drives have a modular design that allows the converter to be flexibly configured. IFs consist of active power modules that provide power to the intermediate circuit (DC link) and energy recovery when the engine is running in the generator mode, and the motor modules that provide four quadrant vector motor control. Active power modules provide an adjustable DC voltage that remains unchanged when the mains voltage changes within the allowed tolerances, in addition active network modules consume almost sinusoidal current from the network and practically do not adversely affect the supply network.

\section{Automated control system for mine hoisting machine}


Mine hoisting machines must be equipped with a modern system of automated control of the mine hoisting machine built on the basis of modern programmable logic controllers (PLCs), and ensuring a high level of safety and reliability of the lifting system.

Structurally, the SAC MHE consists of two channels connected by buses. Each channel is an independent PLC with connected input / output devices, sensors and controls. When executing the control program, the PLC analyzes the signals from the sensors and controls, and generates the signals for controlling the lifting equipment. To implement the dualchannel concept, the critical parameters of the lifting system are monitored by both channels using a double set of sensors. In this case, the signals received from the sensors, as well as the control signals generated in the first and second channel, are subject to equivalence control. Thus, the control of the serviceability of both channels of the SACSH is performed.

A distinctive feature of the proposed system of technological automation is the use of the newest Simatic S7-1500 PLCs, the production of which was started by Siemens in 2013. The PLC of the Simatic S7-1500 family comes to replace the Simatic S7-300 and Simatic PLC families manufactured since the mid-1990s S7-400. Due to the fact that the standard lifetime of the lifting installation is 25 years, the use of the Simatic S7-400 family in the PLC, which are now obsolete and creates significant difficulties in terms of providing spare parts in the operation of the ship.

The structure of SACSHPM includes the following devices [3, 4]:

- Lift control cabinet (PCU) in which the PLCs of channel A, PLC B of channel B, and the parameter recorder are located;

- Shield of the drives (SHCHV), in which the starting and protective equipment and uninterruptible power supplies are located;

- I / O station (SVB) for connecting sensors and actuators located in the computer room;

- the console of the mine hoist operator (PM) with the integrated video terminal (VTS) integrated into it;

- engineering station (IS);

To obtain the current parameters of the lifting installation, a set of sensors is connected to the control system, including:

- impulse sensors installed on the shaft of the lifting machine;

- installed in the barrel track sensors;

- temperature sensors installed in the windings of the motor and power transformers, as well as in bearing bearings.

SACSHPM manages the following systems of the lifting machine [3]:

- drive system;

- Brake control system;

- an automated system of trunk signaling and communication;

- complete switchgear.

Each channel SACMHE is made on the basis of the programmable controller Simatic S71500 made by Siemens.

In addition, the SACSH can exchange information with an automated dispatch control system (ASODU).

Lift control cabinet

The lift control cabinet performs the functions of interlocking control of the main drive, brake and all other mechanisms of the lifting installation, as well as performing monitoring and protection functions. The following are the main functions of control, monitoring and protection $[5,6]$ :

- input and storage in the parameters of the lifting system (depth of rise, coordinates of the location of track sensors, etc.), necessary for setting up the control system;

- input and storage of working and protective diagrams for different operating modes;

- Calculation of the task for the speed of rotation of the electric drive; 
- electric drive control;

- brake control;

- monitoring of the current speed by means of two independent channels and two independent speed sensors;

- determination of the current position of the vessels according to the indications of the pulse counters of two independent channels and the path sensors connected to each of the channels; - continuous comparison of the actual values of the position velocity determined in each of the channels, among themselves;

- automatic deceleration call signaling the beginning of the deceleration period;

- overspeed protection;

- protection against over lifting;

- protection against backing,

- control of the rope inlet;

- control of wire rope sagging;

- other protection and interlocks, conditioned by the requirements of the "Safety rules for mining and processing of solid minerals" and the specifics of the equipment;

- formation of messages about the operation of protection or violation of control functions.

Function of setting and controlling the parameters of the movement of the lifting machine.

The most important question in the development of a system for controlling the velocity of vessels of the WS is the choice of a rational structural diagram of the ACS. ACS SHPM, as a rule, are built on a two-circuit or three-loop subordinate control system, with an external speed control loop and internal circuits for regulating current (torque) and voltage. In the three-circuit systems, in the case of the error on the way during the deceleration period of the ship, the fourth contour of the regulation of the position of the vessels of the SHM is additionally connected $[7,8]$.

The functions of setting and controlling the movement of the lifting machine are realized by special program blocks executed in the PLC of channel A and channel B of the automatic control system.

The motion reference function generates a speed and direction reference for the drive of the lifting machine and controls the service brake.

The value of the speed reference is defined as the smaller of the value determined by the tachogram of motion (a set of parameters that determine the speed of the lifting vessel as a function of the position in the trunk) and the command from the commander of the stroke.

SACSHPM allows you to flexibly configure the tachogram motion for the specific conditions of the trunk, taking into account the number of horizons, limiting the speed of passing intermediate horizons, etc. For each mode of operation ("Cargo", "People", "Revision" "Ungulate" "Permutation", etc.), a separate tachogram of movement can be formed.

The parameters of the motion tachograms are stored in the non-volatile memory of the PLC of channel A and channel B.

The value of the speed and direction of motion set in rope A and channel B is subject to equivalence control. In the case of a mismatch, a job formation error is generated.

The configuration of the motion tachograms is performed by the operational personnel, who have the highest priority of the password access to the system configuration of the SAUSH. It is produced using the "man-machine" interface of the SAUSHM system, which simplifies the operation of the operating personnel when entering the barrel parameters. The mutual control of the entered data guarantees the correctness of the entered values, excluding the error when entering.

An example of the screen for monitoring and configuring the motion tachogram is shown in Fig.2. 


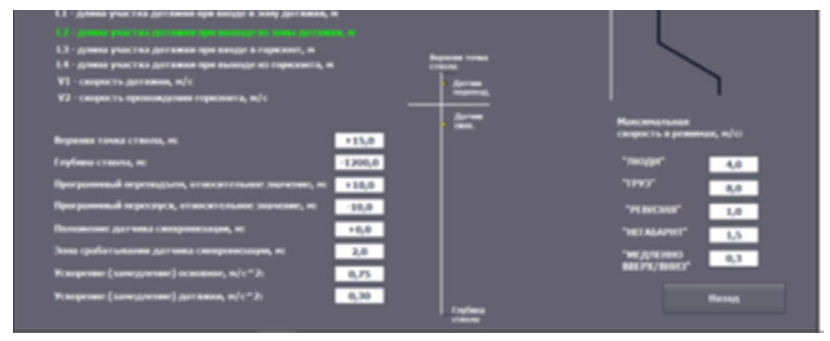

Fig. 2. An example of the screen for configuring the motion tachogram.

Function of monitoring the position of lifting vessels. SAUSHM determines the current position of the lifting vessels by counting the number of pulses received from the incremental encoders mechanically connected to the shaft of the lifting machine. In order to minimize the error caused by wire rope stretching and to avoid the accumulation of errors during operation, the value of the position counter corrects each lifting cycle when the magnetic synchronizing switch installed in the barrel is triggered and triggered every time an lifting vessel passes it.

The value of the current position of the lifting vessels is used to form the motion reference and to implement the redundant (software) protection against over lifting and overrun.

The PLC of channel A and channel B, using two incremental encoders, calculate the positions of the lifting vessels independently of each other. The calculated values of the positions are subject to equivalence control and, in the event that the error exceeds the allowable value, a position monitoring error is generated.

Over-speed protection.

The actual speed of the lift machine is determined by each of the PLCs of channel A and channel B and, in case the speed exceeds the value set by the tachogram of the movement by $15 \%$ (the value is specified during adjustment), an overspeeding error is created, followed by the application of a safety brake. Since SACSHPM is two-channel, it provides full selfmonitoring of the elements involved in limiting the speed of the lifting machine, the use of a duplicating speed limiter is not required.

Two-channel principle of construction SACSHPM guarantees the highest degree of safety carried out with the help of a lifting machine, since both the program and motion control software modules executed in the A channel PLC and the B channel of the SACSHP have all the information about vessel motion, condition and operation modes of the lifting installation. Speed and acceleration (deceleration) are monitored dynamically all along the barrel, which allows to diagnose malfunctions in the drive system, for example, in acceleration and deceleration sections.

\section{Conclusions}

Thus, the system for automated control of the mine hoisting machine (SAUSHM) based on the Simatic S7-1500 PLC, with the developed control algorithm, is an integrated energyefficient control system, which it is advisable to use in the management of the SHM.

\section{References}

1. I. Ya. Braslavsky, Energy-saving asynchronous electric drive (Academy, Moscow, 2004)

2. N. M. Ulaschik, Power conversion technology: a summary of lectures (Publishing Center BNTU, Minsk, 2010) 
3. B. K. Bose, Modern power electronics and AC drives (University of Tennessee, Knoxville, 2002)

4. L. Zhang, Modeling and Control of VSC - HVDC Links connected to Weak AC Systems (Royal Institute of Technology, Stockholm, 2010)

5. E. V. Pugachev, Market review of frequency - regulated electric drives with a link of electric power recuperation into the power supply network (SibGIU, Novokuznetsk, 2014)

6. M. V. Lazareva, Analysis of diagrams of acceleration, jerk and effort in mine hoisting installations (DonNU, Donetsk, 2010)

7. H. Miranda, P. Cortes, J.I. Yuz, IEEE Trans. Ind. Electronics. 6, 1916-1924 (2009)

8. D. Casadei, G. Serra, A. Tani, and L. Zarri, Electromotion Journal, 6, 43-48 (1999) 